\title{
Time-dependent patterns in quasivertical cylindrical binary convection
}

\author{
Arantxa Alonso, ${ }^{*}$ Isabel Mercader ${ }^{\dagger}$ and Oriol Batiste ${ }^{\ddagger}$ \\ Departament de Física, Universitat Politècnica de Catalunya, Mòdul B4, 08034 Barcelona, Spain
}

(Received 31 July 2017; revised manuscript received 26 January 2018; published 26 February 2018)

\begin{abstract}
This paper reports on numerical investigations of the effect of a slight inclination $\alpha$ on pattern formation in a shallow vertical cylindrical cell heated from below for binary mixtures with a positive value of the Soret coefficient. By using direct numerical simulation of the three-dimensional Boussinesq equations with Soret effect in cylindrical geometry, we show that a slight inclination of the cell in the range $\alpha \approx 0.036 \mathrm{rad}=2^{\circ}$ strongly influences pattern selection. The large-scale shear flow (LSSF) induced by the small tilt of gravity overcomes the squarelike arrangements observed in noninclined cylinders in the Soret regime, stratifies the fluid along the direction of inclination, and produces an enhanced separation of the two components of the mixture. The competition between shear effects and horizontal and vertical buoyancy alters significantly the dynamics observed in noninclined convection. Additional unexpected time-dependent patterns coexist with the basic LSSF. We focus on an unsual periodic state recently discovered in an experiment, the so-called superhighway convection state (SHC), in which ascending and descending regions of fluid move in opposite directions. We provide numerical confirmation that Boussinesq Navier-Stokes equations with standard boundary conditions contain the essential ingredients that allow for the existence of such a state. Also, we obtain a persistent heteroclinic structure where regular oscillations between a SHC pattern and a state of nearly stationary longitudinal rolls take place. We characterize numerically these time-dependent patterns and investigate the dynamics around the threshold of convection.
\end{abstract}

DOI: 10.1103/PhysRevE.97.023108

\section{INTRODUCTION}

The study of convective flows in binary mixtures plays an important role in many natural phenomena and engineering applications. Double-diffusive instabilities induced by the interaction of two fields that diffuse at different time rates are behind many noteworthy phenomena in geophysics [1], astrophysics [2], and, especially, in oceanography [3-6]. Driven by the difference in the molecular diffusivities of heat and salt, the double diffusion of salinity and temperature contributes to mixing and induces interesting flow phenomena in the ocean, such as thermohaline staircases and salt fingers (see Radko [7] for a detailed review of the role of double-diffusive convection in oceanography). Examples of industrial applications include crystal growth processes [8] and solar ponds [9].

The combined effects of thermal and solutal buoyancy forces lead to complex flow structures that are also very interesting from a fundamental fluid dynamics and bifurcation theory perspective. Many theoretical and numerical studies on this topic, though, are concerned with the double diffusive problem, in which the flows induced by the buoyancy forces result from the external imposition of both thermal and solutal boundary conditions in the absence of cross-diffusion. However, the dynamics is especially interesting in the case of binary mixtures, in which the generation of concentration fluxes by temperature gradients, known as the Soret effect, gives rise

\footnotetext{
*arantxa@fa.upc.edu

${ }^{\dagger}$ maria.isabel.mercader@upc.edu

${ }^{\ddagger}$ oriol@fa.upc.edu
}

to a variety of convection patterns. Thus, the components of miscible ordinary two-component mixtures such as wateralcohol, normal $\mathrm{He}^{3}-\mathrm{He}^{4}$ mixtures or salt-water mixtures, or the components of colloidal fluids, such as ferrofluids [10], tend to separate in an imposed thermal gradient, and this separation in turn alters the driving force for convection. The Soret coefficient $S_{T}$ measures the strength of the Soret coupling, and its sign determines the behavior of the mixture. With $S_{T}>0$, the heavier component of the fluid is driven into the direction of lower temperature, while with $S_{T}<0$, the heavier component migrates towards the hotter boundary. The driving mechanisms in binary convection are controlled by two nondimensional numbers: the Rayleigh number Ra, measuring the applied temperature stress, and the separation ratio $S$, proportional to $S_{T}$, and quantifying the solutal driving.

In this work we are concerned with thermal convection in a binary fluid layer heated from below, which is a prominent example of a macroscopic dissipative system that undergoes a great variety of pattern forming instabilities when driven away from equilibrium by external stresses [11-13]. We will analyze the behavior of mixtures with $S_{T}>0$, for which the solutal density variations enhance the thermal density variations and further destabilize the fluid layer heated from below. Since the concentration gradient also contributes to the convective behavior, the onset of convection takes place for smaller heating than in the pure fluid case. Experiments on vertical cylindrical cells performed in the 1980s [14,15] revealed that near onset the motion is dominated by concentration gradients (Soret regime), far from threshold convection selects structures observed in pure fluid convection (Rayleigh regime), and in the crossover region complex time-dependent dynamics arises. 
Direct numerical simulations in periodic rectangular cells, and few-mode weakly nonlinear models, provided the phase diagrams of the stable convective states (stationary squares, rolls, crossrolls, and oscillatory crossrolls) that organize the dynamics [16-18]. Although there have been previous threedimensional (3D) numerical simulations in cylindrical cells with vertical heating in pure fluids (e.g., Refs. [19-22]), in binary mixtures they are scarce and, to our knowledge, mainly limit to $S_{T}<0$ mixtures [23,24].

The aim of the present work is to analyze the effect of slightly tilting the cell an angle $\alpha$ on the stability of the previous patterns. Tilting a cavity is a simple and intrinsic way of introducing a shear mechanism in the convection layer. The shear breaks the rotational isotropy of the layer and generates a large-scale circulatory shear flow (LSSF). An interplay with the double-diffusive convective mechanisms is going to take place. The resulting vertically sheared motion will differentially advect properties across lateral fronts and the mixing and transport properties (heat, momentum), are going to be affected. Determining to what extent the dynamics is modified when a convective layer is tilted is an important issue to many practical applications, since convective systems are often inclined with respect to gravity in nature. Even when the cavity is intended to be placed horizontally, a very slight inclination exists in a real physical situation.

In spite of the importance of natural convection in tilted cavities, previous numerical works on binary mixtures are scarce and mostly limited to the linear regime and primary bifurcations in two dimensional or extended configurations. The effect of inclination on three-dimensional (3D) convection in a cylindrical thermal diffusion cell (a laterally heated cell designed to measure the Soret coefficient) was studied numerically in Ref. [25], with the aim of modeling a spatial (microgravity) environment. Many of the interesting effects in Soret-induced convection originate from the difference in relaxation times between the temperature and the concentration field due to the sharp contrast between thermal and solutal diffusivities, but this makes simulations much more costly.

Very recent three-dimensional numerical works deal with the study of inclined layer convection in one-component fluids. Subramanian et al. [26] analyze in extended layers the rich variety of spatiotemporal patterns that had been observed experimentally by Daniels et al. $[27,28]$ when varying the inclination angle. Torres et al. [29] study the transition from the Rayleigh-Bénard convection to the heated-from-the-sides configuration in parallelepipedic cavities. Both works cover a wide range of inclination angles, from quasi-Rayleigh-Bénard convection to the heated from the side configuration. In cylindrical geometry, a pick of the convective patterns arising in a tilted cylinder, for much larger heating and angles also covering the Rayleigh-Bénard case and vertical convection is shown in Ref. [30].

Among the relevant numerical works in inclined 2D binary convection, an analytic and numerical study of the natural convection in an inclined shallow cavity is presented in Ref. [31], where bifurcation diagrams for the onset of convection with different boundary conditions are computed. Other 2D numerical works in tilted cavities restrict to the particular case where the opposing thermal and solutal buoyancy effects are of equal

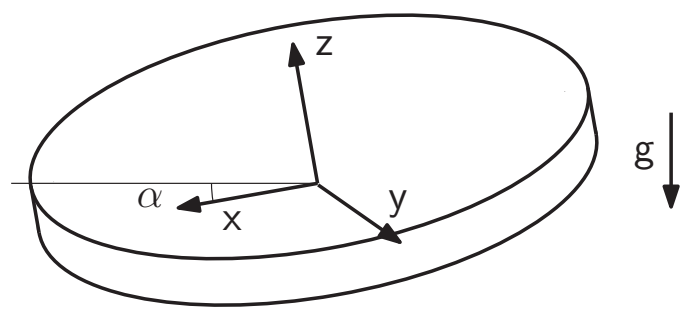

FIG. 1. Sketch showing the geometry of the cell and the choice of axis orientation. The component of gravity along the inclination of the cell, $g_{x}$, is much smaller than the vertical component, $g_{z}$.

magnitude, in which the system admits a conductive basic state [32,33].

Very recently, an outstanding experimental work on slightly inclined binary convection in $S_{T}>0$ mixtures reports timedependent convective patterns that differ substantially from the square, roll, and cross-roll patterns observed in noninclined binary convection [34]. Some unexpected results are obtained: Pattern formation seems to be strongly affected by marginal inclinations and complex time-dependent states exhibiting flow motion in opposite directions along parallel lanes, named superhighway convection (SHC) by the authors, have been observed in the Soret regime. Motivated by this work and using the same fluid parameter values as those of the experiment, we seek to obtain numerically and characterize the patterns observed in the experiment and explore the dynamics for moderate values of the external heating $\mathrm{Ra}$ and small inclinations of the cell. Numerical simulations are a very useful tool to deepen in the knowledge of the underlying dynamics, since they allow to overcome some of the limitations of the experiment, such as the fact that optical measurements could only be obtained in the central part of the cell [34].

The organization of the paper is as follows. In Sec. II, we formulate the equations, boundary conditions, and symmetries of the system, and we explain the numerical methods used. In Sec. III, the main results are discussed: the properties of the LSSF, of the state of SHC, and of a complex state involving a heteroclinic connection between the periodic SHC state and the stationary longitudinal roll state (SHC-roll) are discussed in Secs. III A, III B, and III C, respectively. Finally, we summarize and outline the relevance of our results in Sec. IV.

\section{FORMULATION OF THE PROBLEM: EQUATIONS, SYMMETRIES AND NUMERICAL METHODS}

We consider Boussinesq binary-fluid convection in a cylindrical cell of height $H$ and radius $R$, slightly inclined an angle $\alpha$ with respect to the horizontal. The cylinder is heated from below, $\Delta T$ being the temperature difference between the lids. The $z$ axis is taken along the axis of the cylinder, the origin is located in the hotter lid, and the $x$ axis is chosen in such a way that the gravity is contained in the $x-z$ plane (see Fig. 1). Thus,

$$
\mathbf{g}=g \sin \alpha \hat{\mathbf{e}}_{x}-g \cos \alpha \hat{\mathbf{e}}_{z} .
$$

We split the temperature, $T$, and concentration of the denser component, $C$, fields in a linear profile in $z$ and fluctuations 
$\Theta^{*}$ and $\Sigma^{*}$

$$
\begin{aligned}
& T=T_{0}+\Delta T\left(\frac{1}{2}-\frac{z}{H}\right)+\Theta^{*}, \\
& C=C_{0}-C_{0}\left(1-C_{0}\right) S_{T} \Delta T\left(\frac{1}{2}-\frac{z}{H}\right)+\Sigma^{*},
\end{aligned}
$$

where $T_{0}$ and $C_{0}$ are the mean values and $S_{T}$ is the Soret coefficient. With this decomposition the mass flux only depends on the gradient of $\Theta^{*}$ and $\Sigma^{*}$.

Scaling lengths with the height of the cylinder $H$, time with the vertical thermal diffusion time $H^{2} / \kappa, \kappa$ being the thermal diffusivity, temperature with $\Delta T$ and concentration with $-C_{0}\left(1-C_{0}\right) S_{T} \Delta T$, inclined binary fluid convection is described by the following nondimensional equations:

$$
\begin{aligned}
\nabla \cdot \mathbf{u}= & 0 \\
\partial_{t} \mathbf{u}+(\mathbf{u} \cdot \nabla) \mathbf{u}= & -\nabla P+\sigma \nabla^{2} \mathbf{u}+\operatorname{Ra}_{z} \sigma(\Theta+S \Sigma) \hat{\mathbf{e}}_{z} \\
& -\operatorname{Ra}_{x} \sigma(\Theta+S \Sigma) \hat{\mathbf{e}}_{x} \\
& +\operatorname{Ra}_{x} \sigma(1+S)\left(z-\frac{1}{2}\right) \hat{\mathbf{e}}_{x} \\
\partial_{t} \Theta+(\mathbf{u} \cdot \nabla) \Theta= & w+\nabla^{2} \Theta \\
\partial_{t} \Sigma+(\mathbf{u} \cdot \nabla) \Sigma= & w+\tau\left(\nabla^{2} \Sigma-\nabla^{2} \Theta\right)
\end{aligned}
$$

where $\mathbf{u}$ denotes the nondimensional velocity field, $w$ its $z$ component, $P$ is the nondimensional kinematic pressure plus the contributions of the gravitational force that can be written as a gradient, $\Theta$ the nondimensional fluctuation of the temperature and $\Sigma$ stands for the rescaled fluctuation of the concentration.

The system is specified by the inclination angle $\alpha$ and four dimensionless parameters: the Rayleigh number Ra that provides a dimensionless measure of the vertical imposed temperature difference $\Delta T$, the separation ratio $S$ that measures the concentration contribution to the buoyancy force due to cross-diffusion, and the Prandtl and Lewis numbers $\sigma, \tau$, in addition to the aspect ratio $\Gamma=R / H$. These parameters are defined as follows:

$$
\begin{aligned}
\mathrm{Ra} & =\frac{\gamma g \Delta T H^{3}}{\kappa \nu}, \quad S=C_{0}\left(1-C_{0}\right) \frac{\beta}{\gamma} S_{T}, \\
\sigma & =\frac{\nu}{\kappa}, \quad \tau=\frac{D}{\kappa},
\end{aligned}
$$

where $\gamma$ and $\beta$ are the thermal and concentration expansion coefficients, $D$ is the mass diffusivity, and $v$ is the kinematic viscosity. In the equations $\mathrm{Ra}_{x}=\mathrm{Ra} \sin \alpha$ and $\mathrm{Ra}_{z}=$ $\mathrm{Ra} \cos \alpha$. More details about the obtention of these equations can be found in some previous works in related configurations $[35,36]$.

We consider impermeable and nonslip boundary conditions, with fixed temperature at the lids and insulating boundaries on the lateral wall:

$$
\begin{aligned}
& \mathbf{u}=\Theta=\partial_{z}(\Sigma-\Theta)=0 \quad \text { on } z=0,1, \\
& \mathbf{u}=\partial_{r} \Theta=\partial_{r}(\Sigma-\Theta)=0 \quad \text { on } r=\Gamma .
\end{aligned}
$$

As a measure of the strength of convection we will use the dimensionless mean kinetic energy defined as

$$
E=\frac{\int_{0}^{1} \int_{0}^{2 \pi} \int_{0}^{\Gamma} \mathbf{u} \cdot \mathbf{u} r d r d \theta d z}{2 \pi \Gamma^{2}} .
$$

The previous system of nondimensional equations (2) are written in cylindrical coordinates $(r, \theta, z)$ as follows:

$$
\begin{aligned}
\nabla \cdot \mathbf{u}= & 0, \\
\partial_{t} u+[(\mathbf{u} \cdot \nabla) \mathbf{u}]_{r}= & -\partial_{r} P+\sigma\left[\nabla^{2} \mathbf{u}\right]_{r}-\operatorname{Ra}_{x} \sigma[(\Theta+S \Sigma) \\
& \left.-(1+S)\left(z-\frac{1}{2}\right)\right] \cos \theta, \\
\partial_{t} v+[(\mathbf{u} \cdot \nabla) \mathbf{u}]_{\theta}= & -\frac{1}{r} \partial_{\theta} P+\sigma\left[\nabla^{2} \mathbf{u}\right]_{\theta}+\operatorname{Ra}_{x} \sigma[(\Theta+S \Sigma) \\
& \left.-(1+S)\left(z-\frac{1}{2}\right)\right] \sin \theta, \\
\partial_{t} w+[(\mathbf{u} \cdot \nabla) \mathbf{u}]_{z}= & -\partial_{z} P+\sigma\left[\nabla^{2} \mathbf{u}\right]_{z}+\operatorname{Ra}_{z} \sigma(\Theta+S \Sigma), \\
\partial_{t} \Theta+(\mathbf{u} \cdot \nabla) \Theta= & w+\nabla^{2} \Theta, \\
\partial_{t} \Sigma+(\mathbf{u} \cdot \nabla) \Sigma= & w+\tau\left(\nabla^{2} \Sigma-\nabla^{2} \Theta\right) .
\end{aligned}
$$

The system of Eqs. (5) and boundary conditions (3) has been solved numerically using the algorithm described in Ref. [37], which can be summarized as follows. To integrate the equations in time, we use the second-order time-splitting method proposed in Ref. [38] combined with a pseudospectral method for the spatial discretization, Galerkin-Fourier in the azimuthal coordinate $\theta$, and Chebyshev collocation in $r$ and $z$. The radial dependence of the functions is approximated by a Chebyshev expansion between $-R$ and $R$ but forcing the proper azimuthal parity of the variables at the origin $[39,40]$. Steady solutions have been computed with a Newton's method. We have used a first-order version of the time-stepping code described above for the calculation of a Stokes preconditioner that allows a matrix-free inversion of the preconditioned Jacobian needed in each Newton iteration [41]. The corresponding linear system is solved by an iterative technique using a GMRES package [42]. As far as the linear stability analysis of the steady states is concerned, once they have been calculated by the method described before, estimations of eigenvalues and eigenvectors of the linearized problem have been obtained with an Arnoldi's method.

Equations and boundary conditions are equivariant under the group of symmetries $G$ that contains the transformations $\left\{I, R_{1}, R_{2}, R_{3}\right\}$, where $I$ stands for the identity, $R_{1}$ is a reflection with respect to the middle longitudinal vertical plane ( $y=$ $0), R_{2}$ is a point symmetry with respect to the center of the cylinder, and $R_{3}$, which is the composition of the previous transformations, is a rotation by $\pi$ about the line $x=0, z=$ $1 / 2$, the diameter parallel to the $y$ axis located in the center of the cylinder. The $G$ group has three subgroups $\left\{I, R_{1}\right\},\left\{I, R_{2}\right\}$, and $\left\{I, R_{3}\right\}$, which are all different but isomorphic to $Z_{2}$. In addition, $G$ can be generated by combining any two of these three $Z_{2}$ subgroups, so we write $G=Z_{2} \otimes Z_{2} \equiv D_{2}$, where $\otimes$ indicates the direct product of the groups, used when each 
element of either group commutes with every element of the other group.

These transformations act on the cylindrical components of the velocity field and deviation of the temperature $u, v, w$, as follows:

$$
\begin{aligned}
R_{1}:(r, \theta, z) & \rightarrow(r,-\theta, z), \\
(u, v, w, \Theta, \Sigma) & \rightarrow(u,-v, w, \Theta, \Sigma), \\
R_{2}:(r, \theta, z) & \rightarrow(r, \pi+\theta, 1-z), \\
(u, v, w, \Theta, \Sigma) & \rightarrow(u, v,-w,-\Theta,-\Sigma), \\
R_{3}:(r, \theta, z) & \rightarrow(r, \pi-\theta, 1-z), \\
(u, v, w, \Theta, \Sigma) & \rightarrow(u,-v,-w,-\Theta,-\Sigma) .
\end{aligned}
$$

\section{RESULTS}

To perform the simulations discussed in this section we have taken as reference values those of the experiment [34]. The nondimensional parameters for the isobutylbenzene- $n$ dodecane at $50 \%$ weight fraction binary mixture used are $S=0.13, \tau=0.011$, and $\sigma=16$. The value of the aspect ratio of the cell has been reduced from $\Gamma=10$ in the experiment, to $\Gamma=5$ in the numerical simulations. The majority of the results that will be discussed have been obtained with an inclination of $\alpha=0.024 \mathrm{rad}$, one of the values used in the experimental setup. Slight variations of $S, \tau$, and $\alpha$ around the reference values will be considered. Nondimensional time units are used throughout the paper: one nondimensional time unit corresponds to $20 \mathrm{~s}$.

In our simulations we have used 34 collocation points in the vertical direction, 150 Fourier modes in the azimuthal direction, and 100 points in the radial direction. For steady solutions (LSSF) we have used a resolution that ensures variations of the values of Rayleigh number and frequency at the bifurcation points smaller than $0.5 \%$. In time-dependent computations, for this grid size, a time step of $5 \times 10^{-4}$ has proved to be sufficient to achieve convergence. We have made parallel simulations with a finer mesh, and time series follow similar paths during the time of the integration. It is important to emphasize that, due to the small value of the Lewis number $\tau=0.011$, very long integration times are required for the concentration field to establish.

Before showing results with inclination, we briefly discuss the main features of the dynamics in the noninclined cell. Setting $\alpha=0$, we have performed some numerical simulations for the specific parameters of interest in this work. For a $\sigma=16$ pure fluid in a $\Gamma=5$ cell the onset of convection takes place at $\mathrm{Ra}_{c}=1700$. When a positive separation ratio mixture replaces the pure fluid, the heavier component tends to accumulate at the upper cool plate, and this causes a substantial decrease in the convective onset. In particular, our numerical simulations for a $S=0.13, \tau=0.011$ mixture set the threshold of convection at $\mathrm{Ra}_{c}=61.9$. Convection starts very early in the form of irregular large squares and, as Ra increases, the size of the cells decreases and the convective patterns become progressively more ordered until regular quasistationary square patterns are reached. These structures are typical of the Soret regime, in which convection is primarily driven by solutal buoyancy. For our mixture, this regime approximately extends up to
$\mathrm{Ra} \approx 1800$, where the square patterns are replaced by timedependent cross-roll solutions, nearly stationary longitudinal rolls, and convective structures characteristic of the Rayleigh regime, where thermal driving dominates.

\section{A. LSSF state}

When an inclination as small as $\alpha=0.024$ rad is introduced there is no motionless state and as soon as Ra is different from zero a large-scale flow (LSSF) is naturally generated in the cell, due to the interaction of the very small component of the horizontal gravity, $g_{x}$, and the vertical temperature gradient. This circulation flow overcomes completely the square patterns obtained in the Soret regime for the horizontal cylinder. The main features of the LSSF can be visualized in Fig. 2, which corresponds to a solution obtained for $\mathrm{Ra}=1610$. Figure 2(a) shows the $3 \mathrm{D}$ isosurfaces of the deviation of temperature from the vertical linear profile, $\Theta$. Figure 2(b) shows the arrow plots of the velocity field and the contour plots of $\Theta$ and $C$ in a vertical plane along the direction of inclination. Figure 2(c) shows the $u-v$ velocity field at the $z=0.8$ plane and the Figs. 2(d) and 2(e) the contour plots of $\Theta$ and $C$ at the midplane of the cell $(z=0.5)$. Throughout the paper, to represent the temperature field we display the deviation from the vertical linear profile $\Theta$, while for the concentration field we show the total concentration $C$ of the denser component rather than its deviation from the vertical linear profile $\Sigma$. Also, in the colormap used to represent the contour plots, yellow (light) corresponds to the higher values of the field and blue (dark) to the lower values. The LSSF shares the three symmetries of the system and, as expected, is an essentially horizontal circulatory shear motion of the fluid layer into opposite directions: the warm fluid near the lower lid of the cylinder moves horizontally uphill (to negative $x$ ), parallel to the lid, and when arrives to the lateral boundary, it flows vertically upwards generating a narrow boundary layer; then, the fluid moves horizontally downhill (to positive $x$ ), and flows vertically downwards in the lower part of the cylinder. The temperature variations from the vertical linear profile $\Theta$ are very small outside the lateral boundaries so, away from the boundary layers, $\Theta$ is approximately zero in any horizontal plane [Fig. 2(d)]. However, this is not the case for the concentration field, and a nearly linear horizontal concentration profile is established across the cylinder. The mechanism is as follows. In the vertical direction, since $S>0$, the heavier component of the mixture diffuses towards the cooler region causing a vertical stratification, with concentration accumulated near the top boundary of the cylinder. In the horizontal direction, the large-scale base flow, which is directed down the slope along the top boundary, advects concentration to the less elevated part of the cylinder. This leads to a substantial increase in $C$, and a corresponding deficit in the more elevated part. A slow but quite important separation of the mixture along the horizontal $(z=\mathrm{cnt})$ planes takes place and a roughly uniform horizontal stable concentration gradient is established across the cylinder [Fig. 2(e)].

A linear stability analysis of the LSSF reveals that this basic flow undergoes a Hopf bifurcation at $\mathrm{Ra}_{c}=1695$ that breaks the $R_{2}$ and $R_{3}$ symmetries and preserves the $R_{1}$ left-right symmetry. The critical frequency is $\omega_{c}^{\mathrm{LSSF}}=2.19$, and the 


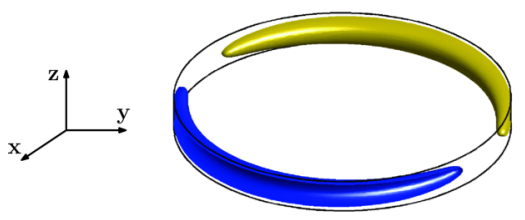

(a)

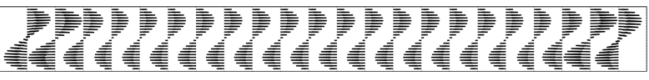

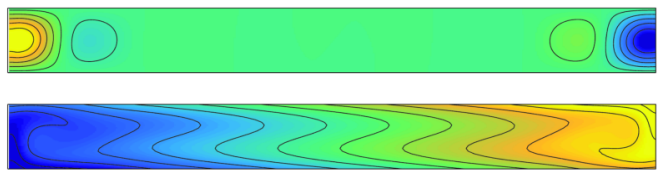

(b)

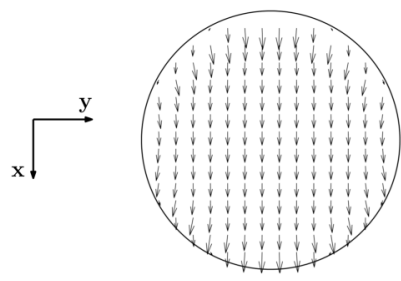

(c)

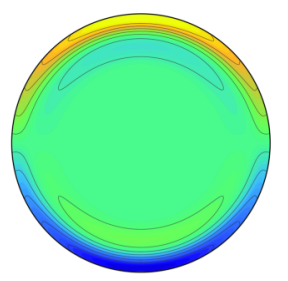

(d)

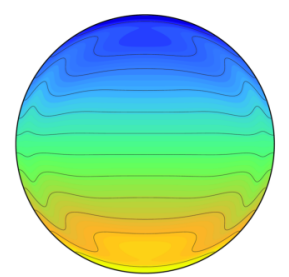

(e)

FIG. 2. The LSSF obtained for $\mathrm{Ra}=1610$ and $\alpha=0.024 \mathrm{rad}$. (a) Isosurfaces of temperature deviation from a vertical linear profile, $\Theta$. (b) Arrow plot of the $u-w$ velocity field (top panel), contour plots of constant temperature fluctuation $\Theta$ (middle panel), and contour plots of concentration of the denser component $C$ (lower panel) in the vertical plane $y=0$ along the direction of inclination of the cell. In these views, the more elevated part of the cell is on the left. (c) Arrow plot of the $u-v$ velocity field in a horizontal plane at height $z=0.8$. (d) Contour plots of constant temperature fluctuation $\Theta$ at midplane $z=0.5$. (e) Contour plots of constant concentration of denser component $C$ at $z=0.5$. In the colormap used here and throughout the paper yellow (light) corresponds to the higher values of the field and blue (dark) to the lower values.

critical eigenfunction superimposed to the LSSF gives rise to pulsating transverse rolls, perpendicular to the direction of inclination, as can be appreciated in Fig. 3. In time-dependent numerical simulations just above this critical value $\mathrm{Ra}_{c}$ taking as initial condition the steady LSSF flow, pulsating transverse rolls are observed, but do not saturate, and the system evolves towards erratic nonperiodic time-dependent patterns. This is an indication that this bifurcation is subcritical.

\section{B. SHC state}

In this subsection we are going to analyze the peculiar traveling patterns reported in the experiment of Croccolo et al. [34], the so-called SHC states, which consist of drifting
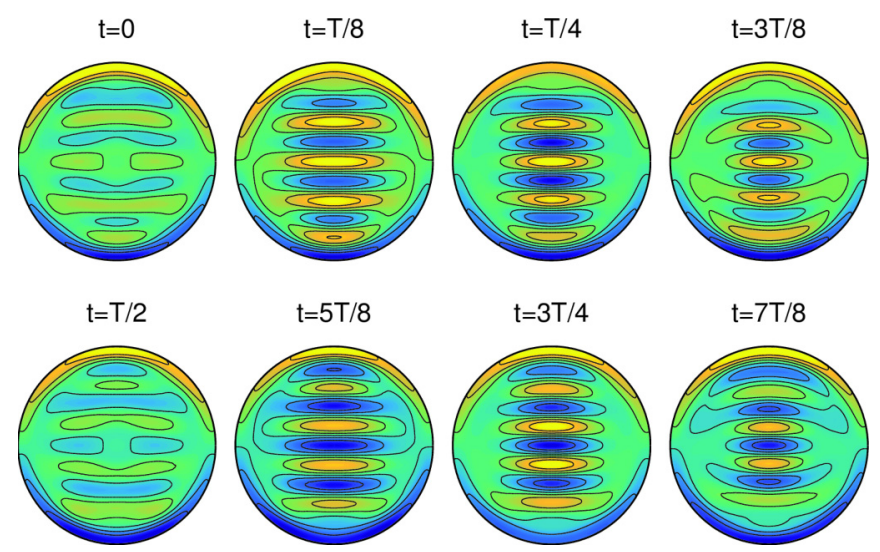

$\mathrm{t}=7 \mathrm{~T} / 8$

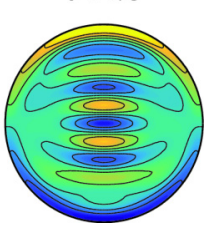

FIG. 3. Contour plots of constant temperature at $z=0.5$ for eight time instants within a period showing the spatiotemporal structure of the superposition of the LSSF and the eigenfunction associated to the pair of leading eigenvalues in the Hopf instability of the LSSF that takes place at $\operatorname{Ra}_{c}=1695$ with $\omega_{c}=2.19$. This bifurcation is subcritical. flow structures moving in opposite directions along parallel lanes. To emulate the experimental procedure, where a typical measurement sequence consists of imposing a vertical temperature difference to a homogeneously mixed sample and observing the evolution of the system, we have integrated the equations taking as initial condition for the time-stepper a nomotion mixed state of uniform temperature and concentration fields. In this way, we have obtained several stable states with a spatiotemporal behavior consistent with the SHC states described in the experiment.

Once we have obtained numerically the SHC state, we can analyze its properties in detail. In Fig. 4 and Fig. 5 we show the main features of a persistent SHC pattern obtained for $\mathrm{Ra}=1610$, which coexists with the stable LSSF solution represented in Fig. 2. Superimposed to the underlying circulatory LSSF, regions of ascending and descending fluid in the vertical direction ( $z$ axis) emerge in the bulk and group within parallel lanes aligned along the direction of inclination ( $x$ axis). This pattern has a specific orientation: each lane, alternately, contains either warm low concentration regions of ascending fluid or cool high concentration regions of descending fluid. In addition, and quite strikingly, these regions do not remain stationary or drift slowly as happens in square patterns arising in noninclined $S>0$ binary convection [14,15,17], but instead they move in the horizontal plane either downhill $(x>0$ direction) if their concentration is higher than that of the environment [corresponding to cooler regions, that is, to blue (dark) zones in the temperature contour plot in Fig. 4(d)], or uphill $(x<0$ direction) if their concentration is lower (corresponding to warmer regions, that is, to yellow (light) zones in the temperature contour plot).

Table I provides an overview of some characteristic values of the velocity and concentration fields corresponding to the two stable coexisting solutions obtained for the same value of the Rayleigh number, $\mathrm{Ra}=1610$, the LSSF and the SHC patterns. The larger values of the velocity field in the case 


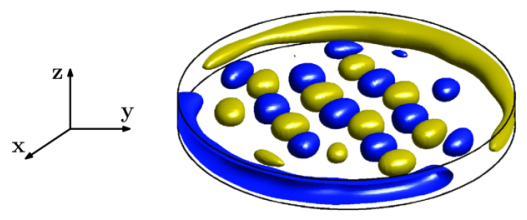

(a)
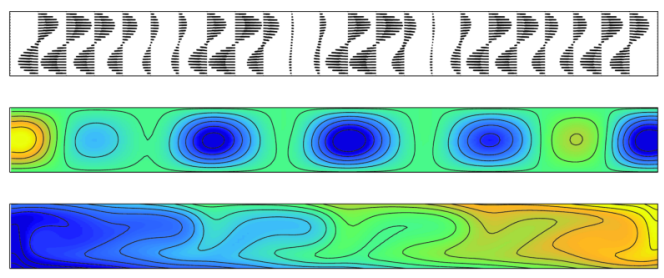

(b)

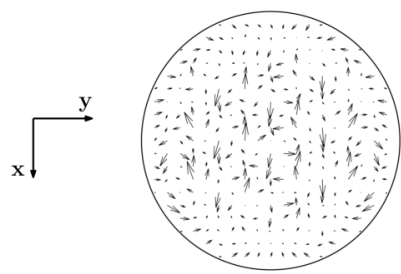

(c)

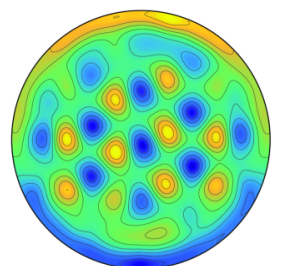

(d)

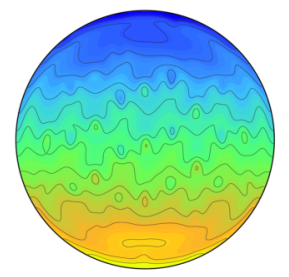

(e)

FIG. 4. The SHC obtained for $\mathrm{Ra}=1610$ and $\alpha=0.024 \mathrm{rad}$. (a) Isosurfaces of constant temperature deviation from a vertical linear profile, $\Theta$. (b) Arrow plot of the $u-w$ velocity field (top panel), contour plots of constant temperature fluctuation $\Theta$ (middle panel) and contour plots of constant concentration of the denser component $C$ (lower panel), in a vertical plane along the direction of inclination of the cell. (c) Arrow plot of the $u-v$ velocity field at midplane $z=0.5$. (d) Contour plots of constant temperature fluctuation $\Theta$ at $z=0.5$. (e) Contour plots of constant concentration of denser component $C$ at $z=0.5$.

of the SHC solution reflect a more vigorous fluid motion than in the LSSF solution. The decrease in the maximum concentration difference across the cylinder in the direction of inclination, $\Delta C^{\max }$, in the SHC solution suggests that the translation motion in opposite directions of the regions with ascending and descending fluid that arises in the bulk of the cell, produces a concentration pumping that results in a slight decrease of the concentration gradient along the incline.

The temperature space-time plots at midplane, $z=0.5$, in Fig. 5 capture the translation motion of the location of the warmer and cooler regions in opposite directions. The top part of Fig. 5(a) shows the variation of $\Theta$ with time in the diameter of the cell along the incline, while the bottom part of Fig. 5(a) shows the space-time plot of $\Theta$ in an off- diameter line, also parallel to the direction of inclination of the cell. While the diameter of the cell is occupied by colder regions of descending fluid with concentration higher than the environment, which are pumped horizontally downhill, the off-diameter line is occupied by hotter regions of ascending fluid with a lower concentration, which are pumped uphill. The time series in Fig. 5(b), which show the the vertical velocity $w$ at $(r, \theta, z)=(4.9,0.04,0.8)$ and the kinetic energy $E$ of the pattern, correspond to a periodic signal and confirm that the SHC pattern is a periodic solution. Its frequency determines the traveling velocity of the regions of ascending and descending fluid in the horizontal direction, and can be obtained from the period of the time series in Fig. 5(b). For Ra $=1610$ this frequency is $\omega=1.508$, and Table II shows the frequency
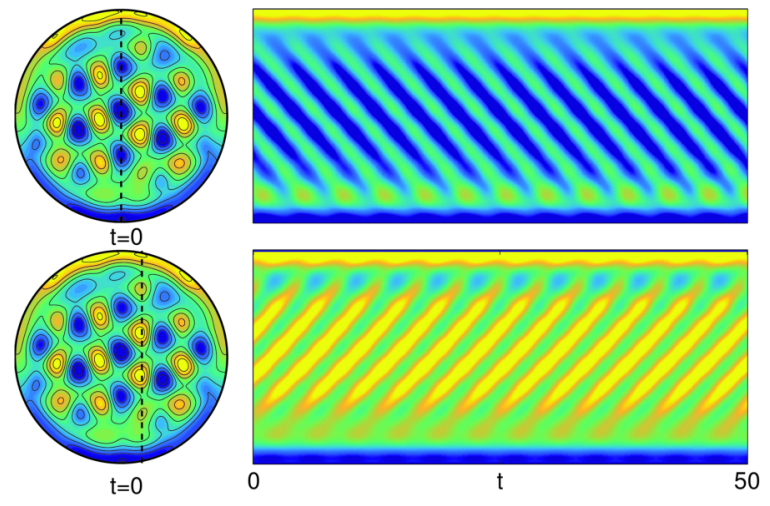

(a)

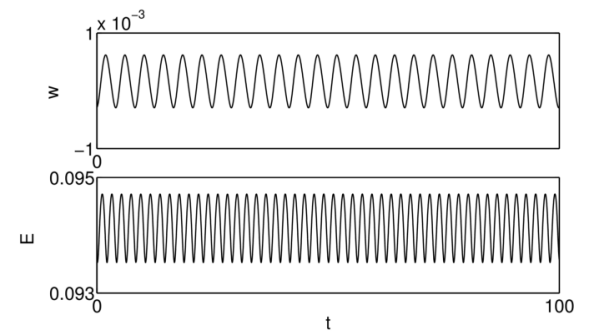

(b)

FIG. 5. The SHC obtained for $\mathrm{Ra}=1610$ and $\alpha=0.024 \mathrm{rad}$. (a) At top, contour plots of constant temperature fluctuation $\Theta$ at $z=0.5$ and $t=0$, and space-time plot showing the evolution of $\Theta$ in a cell diameter parallel to the direction of inclination (dotted line in the contour plot) with time. At bottom, contour plots of constant temperature fluctuation $\Theta$ at $z=0.5$ and $t=0$, and space-time plot showing the evolution of $\Theta$ in an off-diameter line (dotted line in the contour plot) with time. Within each line, the location of the cooler regions of descending fluid forms a wave traveling downhill, while the location of the warmer regions of ascending fluid forms a wave propagating uphill. (b) Time series showing the vertical velocity $w$ at $(r, \theta, z)=(4.9,0.04,0.8)$ and the kinetic energy $E$ of the periodic solution. 
TABLE I. A comparison between a LSSF and a SHC solution, both obtained for $\mathrm{Ra}=1610$. Maximum values of: celerity, $V^{\max }$, horizontal projection of the velocity field, $V_{h}^{\max }$, vertical velocity, $w^{\max }$, vertical velocity at midplane, $\left.w^{\max }\right|_{z=0.5}$, temperature and concentration difference across the cylinder, $\Delta \Theta^{\max }$ and $\Delta C^{\max }$.

\begin{tabular}{lcccccc}
\hline \hline & $V^{\max }$ & $V_{h}^{\max }$ & $w^{\max }$ & $\left.w^{\max }\right|_{z=0.5}$ & $\Delta \Theta^{\max }$ & $\Delta C^{\max }$ \\
\hline LSSF & 0.734 & 0.734 & 0.512 & 0.35 & 0.064 & 1.62 \\
SHC & 1.149 & 0.928 & 1.149 & 0.81 & 0.087 & 1.51 \\
\hline \hline
\end{tabular}

associated to SHC patterns obtained with different values of Ra. Remarkably, this frequency remains approximately constant, $\omega_{\mathrm{SHC}} \approx 1.53$, and reproduces quite accurately the value reported in the experiment, $\omega_{\exp } \approx 1.52$. These observations suggest that in the range of parameters considered in this work the characteristic frequency of a SHC pattern hardly varies with $\mathrm{Ra}$, the vertical heating of the sample, and with the aspect ratio $\Gamma$ of the cell, since the experiment is performed in a $\Gamma=10$ cell while simulations correspond to a $\Gamma=5$ cylinder.

To allow for a closer inspection of the spatiotemporal structure of a SHC pattern, we plot in Fig. 6 contour plots of the temperature field in horizontal planes and, superimposed to them, an arrow representation of the projection of the velocity field at different time instants within a period. Although this solution has also been obtained with $\mathrm{Ra}=1610$, it differs from the SHC pattern shown in Fig. 5 in its transverse wavenumber. While the pattern represented in Fig. 5 has an odd number of lanes (nine rows) and the central diameter along the inclination of the cylinder is occupied with cold regions traveling horizontally downhill, the pattern shown in Fig. 6 contains an even number of lanes (eight rows), with hot regions traveling up the slope at the right-hand side of the central diameter and cold cells traveling down the slope at the left-hand side. Therefore, persistent SHC patterns with a different number of traveling lanes can coexist for the same value of $\mathrm{Ra}$.
TABLE II. Frequency of oscillation of the SHC solution obtained numerically, $\omega_{\text {num }}$, for different values of Ra. This frequency remains approximately constant, $\omega_{\mathrm{SHC}} \approx 1.53$, and matches extremely well the value reported in the experiment, $\omega_{\exp } \approx 1.52$.

\begin{tabular}{lllllll}
\hline \hline Ra & 1575 & 1590 & 1600 & 1610 & 1620 & 1630 \\
\hline$\omega_{\text {num }}$ & 1.508 & 1.571 & 1.571 & 1.508 & 1.532 & 1.508 \\
\hline \hline
\end{tabular}

To gain physical understanding of the SHC state and figure out the motion of the fluid within a period we represent in Fig. 6 isolines of constant temperature at different time instants. In particular, in Fig. 6(a), we represent an arrow plot of the $u-v$ velocity field at midplane at a single time instant, $t=0$, while Fig. 6(b) and Fig. 6(c) contain a detail of the central part of the cylindrical cell at four time instants, $t=$ $0, T / 4, T / 2,3 T / 4$, at $z=0.45$ (below midplane) and at $z=$ 0.55 (above midplane), respectively. The arrow plot of the $u-$ $v$ velocity field at midplane [Fig. 6(a)], where the velocity of the fluid due to the LSSF would vanish, reveals that the fluid moves in opposite directions in alternating lanes. In contrast, the velocity of the fluid below midplane is essentially uphill [Fig. 6(b)], and above midplane essentially downhill [Fig. 6(c)]; that is, the fluid is globally transported by the LSSF. We also observe that, below midplane, the arrows converge in the warmer spots, so the fluid moves from the colder areas towards the warmer ones, while above midplane, the arrows converge in the colder spots, which corresponds to a motion from the warmer areas towards the colder ones. A closer inspection reveals that, any of the cold spots below midplane in the central part of the cell delivers fluid essentially to three neighbor warm spots. More specifically, and alternating twice within a period, the fluid goes from the cold spot to two warm spots on its left and to one on its right $(t=0, T / 2)$ and, after a quarter of period, from the cold spot to two warm spots on its right and to one on its left $(t=T / 4,3 T / 4)$. To complete the picture of the motion of the fluid within a period it is also

(b)
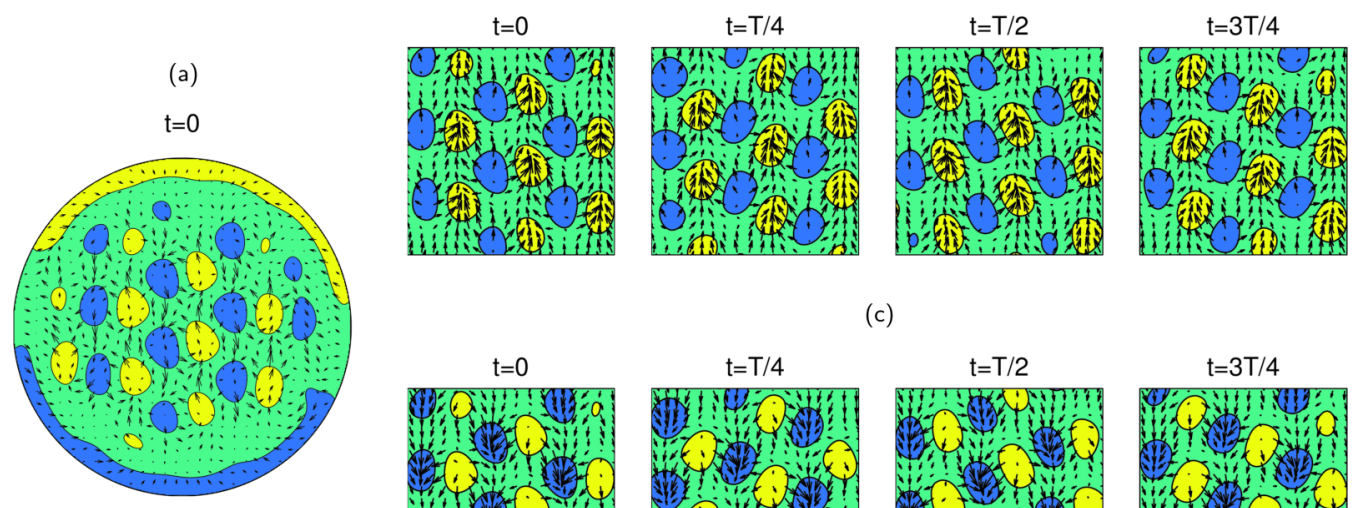

(c)
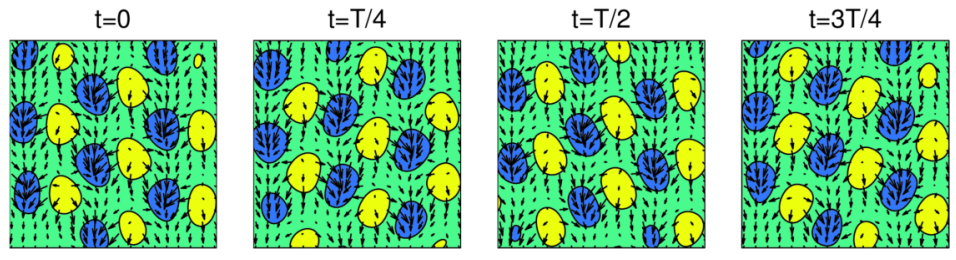

FIG. 6. (a) Contour plot of constant temperature and arrow plot of the $u-v$ velocity field at midplane $z=0.5$ in time instant $t=0$. Detail of the contour plots of constant temperature and arrow plots of the $u-v$ velocity field at (b) $z=0.45$ and (c) $z=0.55$ for four time instants within a period showing the spatiotemporal structure of a SHC state in the central part of the cell. The isolines of temperature are $\Theta= \pm 0.015$, which correspond to $\pm 0.35 \Theta_{\max }$. 
(a)

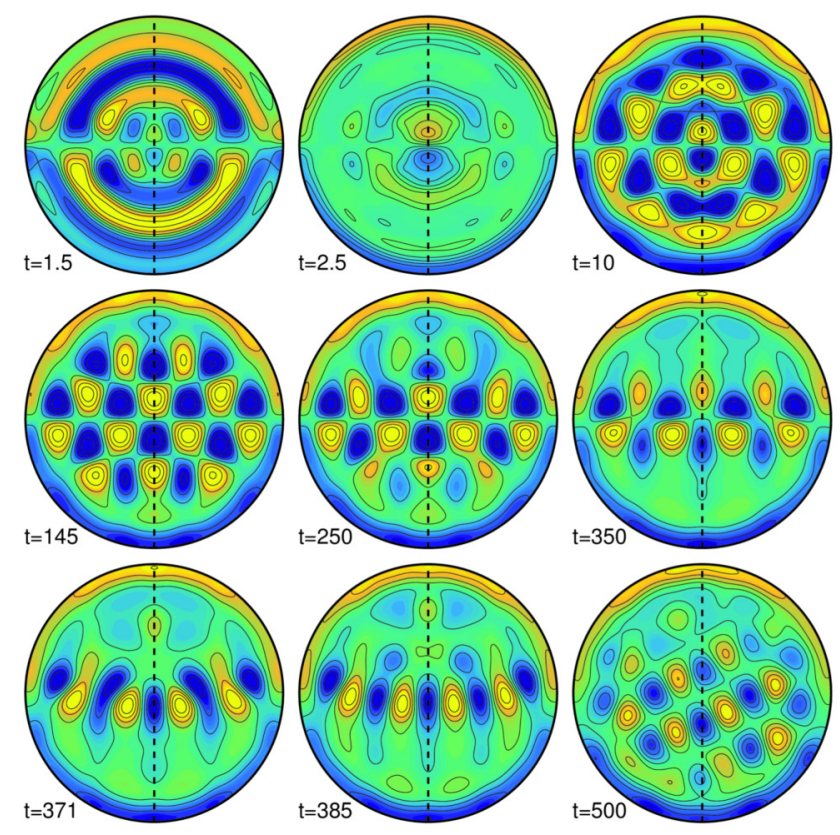

(b)

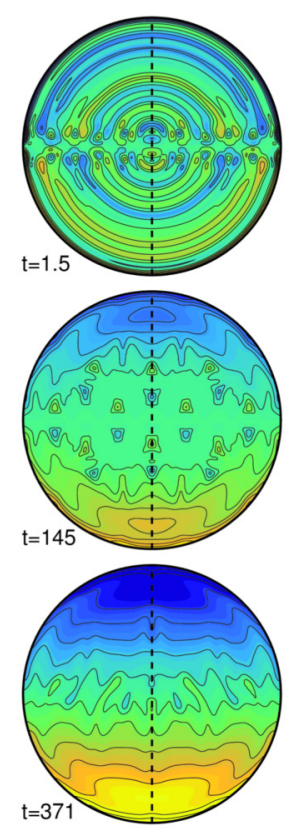

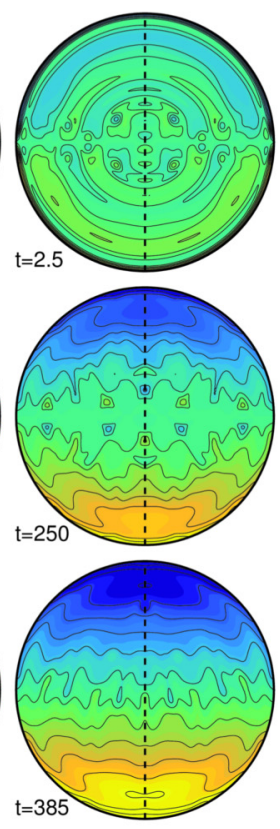

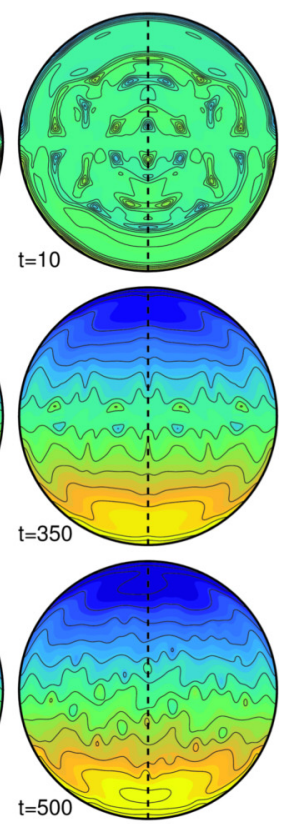

(c)

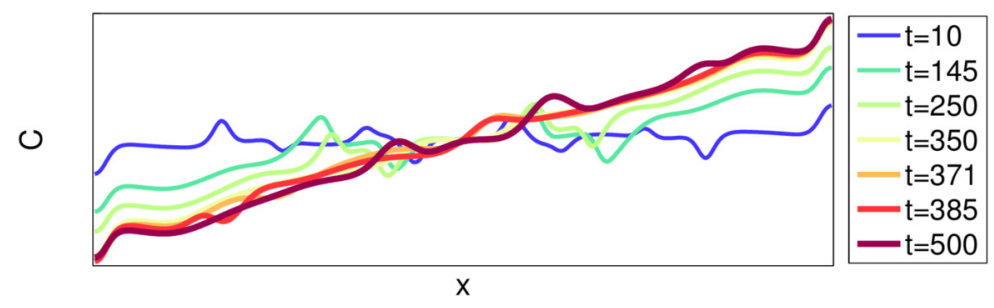

FIG. 7. Contour plots of constant (a) temperature fluctuation $\Theta$ and (b) concentration $C$ at midplane $z=0.5$ showing the formation of a SHC pattern for $\mathrm{Ra}=1610, \alpha=0.024 \mathrm{rad}$, when simulations are initiated with an homogeneous state. The time instants correspond to $t=1.5,2.5,10,145,250,350,371,385,500$ (nondimensional units). (c) Concentration profile at midplane along a cell diameter parallel to the direction of inclination of the cell at the previous time instants. Whereas the circulatory flow is rapidly established in the cell, the horizontal concentration gradient is slowly generated by mass diffusion at a rate two orders of magnitude smaller than heat diffusion.

necessary to focus on the actual location of the cold and warm spots both below and above midplane. We can appreciate that the cold spots translate downhill while the warm spots translate uphill. As a result, as can be clearly visualized in Movie 1 in the Supplemental Material [43], the regions of ascending and descending fluid experience a left-right meandering motion in their displacement along the $x$ direction and a change of shape within a period, due to their oscillating fluid interchange between adjacent lanes. It is worth emphasizing that Fig. 6 reveals that it is the location of the regions of ascending and descending fluid, rather than the fluid itself, what translates uphill and downhill.

To analyze the process of formation of a SHC state, we represent in Fig. 7 a sampling of some of the significant states visited by the system when the mixture is initially well-mixed and a temperature gradient is applied (numerically, we take an homogeneous state as initial condition for the time-integration). Temperature and concentration contour plots at midplane for $\mathrm{Ra}=1610$ at different time instants are shown in Fig. 7(a) and Fig. 7(b), respectively. During the very fast initial linear growth phase, convective patterns develop and change fast. A large-scale circulatory flow, with temperature variations confined to the narrow boundary layers, is rapidly established in the cell and a solution sharing all the symmetries of the system emerges $(t=2.5)$. In a slow, much more ordered second phase, quasistationary square patterns form $(t=10-t=145)$. A careful inspection of the contour plots reveals that these patterns have already broken the $R_{2}$ and $R_{3}$ symmetries, but still keep the $R_{1}$ reflection symmetry along the diameter of the cell. At this stage, the cells are not arranged as in a SHC pattern: Along the diameter of the cell (and in lines parallel to it) warm and cool regions are alternatively found. A reorganization of the pattern is required to reach a SHC state, where only warm or cool regions are found within the same lane. In a third phase, the patterns confine along a diameter of the cell, in a transverse direction with respect to the direction of inclination of the cell $(t=250-t=385)$. After this localization, some oscillations 

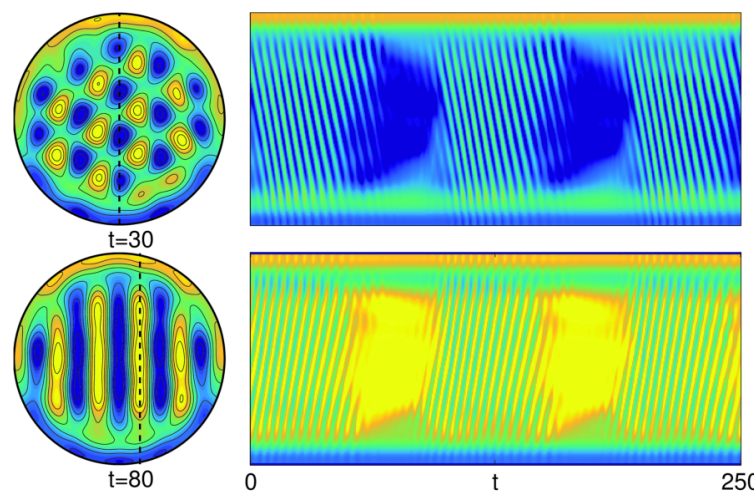

(a)
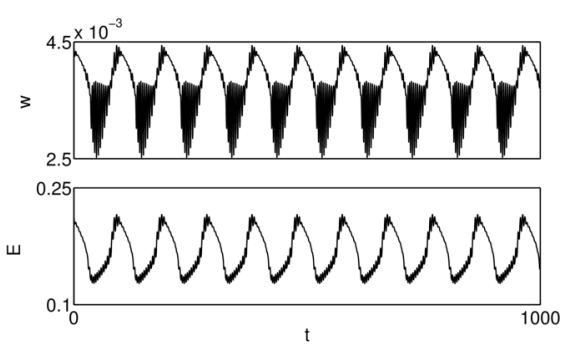

(b)

FIG. 8. Persistent oscillations between a superhighway convection solution and nearly stationary longitudinal rolls (SHC-LHC oscillations) obtained for $\mathrm{Ra}=1680$ and $\alpha=0.024 \mathrm{rad}$. (a) At top, contour plots of temperature fluctuation $\Theta$ at $z=0.5$ and $t=30$, and space-time plot showing the evolution of $\Theta$ in a cell diameter parallel to the direction of inclination (dotted line in the contour plot) with time. At bottom, contour plots of temperature fluctuation $\Theta$ at $z=0.5$ and $t=80$, and space-time plot showing the evolution of $\Theta$ in an off-diameter line (dotted line in the contour plot) with time. The contour plots of $\Theta$ at midplane at two time instants, $t=30$ and $t=80$, show the spatial structure of the two different patterns between which the system oscillates. (b) Vertical velocity $w$ at $(r, \theta, z)=(4.9,0.04,0.8)$ and kinetic energy $E$ of the periodic solution as a function of time $t$.

that seem to initiate in the bulk of the fluid trigger oscillations of the boundary layer. At the final stage, regions of the same type align in lanes parallel to the diameter, and begin to travel in opposite directions: waves are generated and a SHC periodic pattern is established $(t=500)$. Movie 2 in the Supplemental Material [43] shows in more detail the process of formation of the SHC state for $\mathrm{Ra}=1610$. As can be appreciated in the concentration profiles represented in Fig. 7(b), all these warm-cool convective regions are embedded in a horizontal concentration gradient, which is slowly diffusing and takes a long time to establish. To better visualize the growth of this concentration gradient, we plot in Fig. 7(c) the concentration difference in a cell diameter at $z=0.5$ for time instants $t=10,145,250,350,371,385,500$. A minimum value of this concentration difference seems to be required to generate the body force that makes the different lanes travel in opposite directions.

For $S=0.13, \tau=0.011, \sigma=16$, and $\alpha=0.024 \mathrm{rad}$, we have been able to observe such SHC periodic patterns for Rayleigh numbers in the range $\mathrm{Ra} \in(1570,1650)$. However, while for $\mathrm{Ra} \in(1570,1600)$ the SHC solution finally decays away and the only stable solution is the LSSF, for Ra $\in$ $(1600,1650)$ SHC patterns are stable. Thus, the region of stability of the SHC pattern overlaps that of the LSSF solution, which is found to be stable for $\mathrm{Ra}<1695$.

In addition, we have explored the effect on the SHC state of slightly changing the value of the inclination of the cell $\alpha$, the separation ratio $S$, and the Lewis number of the mixture $\tau$. On one hand, we obtain that the frequency of the SHC state, $\omega_{\mathrm{SHC}}$, which determines the velocity of the traveling structures, increases with $\alpha: \omega_{\mathrm{SHC}}(\alpha=0.018)=$ $1.35, \omega_{\mathrm{SHC}}(\alpha=0.024)=1.53$ and $\omega_{\mathrm{SHC}}(\alpha=0.030)=1.69$. However, we do not observe stable SHC patterns for $\alpha>$ 0.034, a fact coincident with experimental observations [34]. On the other hand, increasing the strength of the Soret effect $S$ also increases $\omega_{\text {SHC }}$. For $S=0.20$ we have observed SHC patterns in the range $\mathrm{Ra} \in(1580,1650)$ with a frequency of
$\omega_{\mathrm{SHC}} \approx 1.71$. For this value of $S$, the dynamics becomes faster, i.e., the time required for the formation of a SHC state when starting from an homogeneous state is reduced. Since the horizontal stratification is enhanced when $\alpha$ and $S$ increase, these observations seem to indicate that the large concentration separation across the cylinder induced by the inclination provides the stratified environment that causes the horizontal displacement uphill and downhill of the ascending and descending regions of fluid due to buoyant effects. Finally, increasing the value of $\tau$ also accelerates the dynamics and increases the value of the frequency. For $\tau=0.022$, SHC patterns arise in the range $\mathrm{Ra} \in(1580,1630)$, their frequency being $\omega_{\text {SHC }} \approx 1.74$.

\section{Oscillations between superhighway convection patterns and longitudinal rolls (SHC-LR state)}

When the Rayleigh number is increased above $\mathrm{Ra}=1650$, the SHC pattern becomes unstable and the system evolves towards a nearly stationary configuration: longitudinal rolls aligned along the direction of inclination of the cell superposed to a LSSF (LR). But this state is in turn unstable and the system returns then to the initial SHC state. A long-lived oscillation between these two patterns takes place. Such a state can be envisaged as an heteroclinic connection between two unstable states, the SHC periodic orbit and the stationary longitudinal roll state. We refer to this state as SHC-LR oscillations and its spatiotemporal dynamics can be clearly visualized in Movie 3 in the Supplemental Material [43]. Figure 8 shows the temperature and concentration contour plots at midplane for $\mathrm{Ra}=1680$ as well as the space-time diagram of temperature computed at two parallel lines along the direction of inclination of the cylinder. In pure fluid inclined convection and $\sigma \approx 1$, the longitudinal roll configuration is observed for inclination angles below $78^{\circ}$ [26]. However, in the binary-fluid configuration under consideration, we have not obtained stable stationary longitudinal rolls in the range of Ra explored. Such 
TABLE III. Estimation of the pseudoperiod associated to the SHC-LR oscillations as a function of Ra.

\begin{tabular}{lccccc}
\hline \hline $\mathrm{Ra}$ & 1680 & 1690 & 1700 & 1710 & 1720 \\
\hline$T_{\mathrm{SHC}-\mathrm{LR}}$ & 96.7 & 95.8 & 96.0 & 100.3 & 105.4 \\
\hline \hline
\end{tabular}

a state could emerge for Ra numbers above the ones considered in this work.

We have obtained states of long-lived SHC-LR oscillations in the range $\mathrm{Ra} \in(1650,1700)$. The period associated to this oscillation increases slightly with Ra, as can be seen in Table III, so the time spent by the system visiting each state becomes longer when the thermal forcing increases. Above $\mathrm{Ra}>1700$, the oscillations between these two states disappear completely and the system evolves towards nonoscillating slowly changing patterns. By subsequently increasing Ra we leave the region dominated by solutal effects and enter the thermal regime.

\section{DISCUSSION AND CONCLUDING REMARKS}

The numerical simulations presented in this paper confirm that the intriguing SHC pattern observed recently in the experiments of Croccolo et al. [34] is a periodic orbit of the Boussinesq Navier-Stokes equations for slightly inclined binary convection. We have been able to observe such orbits for Rayleigh numbers in the range $\mathrm{Ra} \in(1570,1650)$. Additionally, in the range $R a \in(1650,1700)$ we have obtained a persistent heteroclinic structure, the SHC-LR state, where regular oscillations between a SHC pattern and a state of nearly longitudinal rolls takes place. The region of stability of these states overlaps that of the LSSF, the basic circulatory large-scale flow established with the inclination of the cylinder, which is stable for $\mathrm{Ra}<1695$. The dynamics we obtain matches extraordinary well the experimental observations, despite the different values of the cell aspect ratio used. Although numerical simulations often oversimplify the fluid physics, and real experiments can easily deviate from the idealized assumptions of numerical studies, in this case the agreement between the experimental observations and the numerical simulations of Oberbeck-Boussinesq Navier-Stokes equations with Soret effect is excellent.

By obtaining numerically the time-dependent patterns that arise in the Soret regime we have been able to characterize them and gain insight in the underlying physical mechanisms that might be relevant in their genesis. The spatiotemporal structure of a SHC pattern could be envisaged as resulting from the interplay of a large-scale shear flow around the cell, which significantly in the case of a binary mixture has an important horizontal concentration gradient, and a configuration of opposite-traveling regions of ascending and descending fluid arranged along parallel lanes in the bulk of the cell that form oscillating asymmetric convective cells. These flows are generated by the combination of buoyant effects in the vertical direction and along the inclination of the cell. In particular, in a $S>0$ mixture, the concentration gradient induced across the cell by its small inclination generates a stable stratified environment along the horizontal direction. When regions of less buoyant fluid (that is, denser fluid regions) generated by the vertical heating encounter this stratified environment they are pumped downhill, towards the less elevated part of the cell; whereas patches of more buoyant fluid (lighter fluid regions) are pumped uphill. However, it is important to notice that the global motion of the fluid is dominated by the large-scale shear flow, and is essentially uphill below midplane and downhill above midplane, with fluid converging to hotter regions below midplane and to colder regions above midplane; it is the location of the hot (cold) spots of ascending (descending) fluid that travels horizontally uphill (downhill) in opposite senses.

Determining the precise origin of the SHC solution would involve obtaining the complete bifurcation diagram of the different solutions observed in the system. This is nonstraightforward task would require the computation of unstable timedependent solutions. The fact that the eigenfunction corresponding to the oscillatory instability gives rise to transverse structures (perpendicular to the direction of inclination) seems to suggest that the SHC solutions do not arise from the subcritical instability of the LSSF, since several subsequent bifurcations from the primary LSSF would be required to reach a SHC solution. Based on the long-lived oscillations that take place between the SHC pattern and the longitudinal rolls, and the spatial similarities between the two patterns (same transverse wavenumber and alignment of ascending and descending fluid regions), we find it more plausible that the SHC pattern may arise from an instability of the longitudinal rolls in the presence of the concentration gradient generated by the LSSF. These conjectures will need to be confirmed in a future work, since, to the authors' best knowledge, not even the bifurcation diagrams describing the square, rolls and crossrolls solution in a noninclined cylinder have been computed to date.

With the present numerical investigation we have provided a useful comparison with the experimental observations of Croccolo et al. [34] and have confirmed the remarkable observation that the smallest tilt in a binary layer under a thermal stress adds an extra body force along the direction of inclination that has a strong influence on the long-term stability of a heated layer. Slightly inclined binary fluid convection in a cylindrical cell under a vertical thermal stress turns out to be a system where the interplay of shear and buoyancy caused by both temperature and concentration variations in a confined geometry self-sustains amazing stable wavelike patterns. This system is well suited for the study of buoyancy- and shear flow-driven instabilities in problems of oceanographic interest and serves as a paradigm for anisotropic pattern forming systems. The results presented in this paper suggest that for multicomponent fluids, such as a binary mixture, the slightest perturbation of a system can produce an enhanced modification of the concentration field which can affect substantially the fluid physics.

\section{ACKNOWLEDGMENTS}

This work has been funded by DGICYT under Grant No. FIS2017-85794-P. We acknowledge A. Vailati and F. Croccolo for their excellent disposition to provide us all the experimental details. 
[1] B. A. Buffett and C. T. Seagle, J. Geophys. Res. 115, B04407 (2010).

[2] T. S. Wood, P. Garaud, and S. Stellmach, Astrophys. J. 768, 157 (2013).

[3] J. S. Turner, Annu. Rev. Fluid Mech. 17, 11 (1985).

[4] R. W. Schmitt, Annu. Rev. Fluid Mech. 26, 255 (1994).

[5] L. H. Kantha and C. A. Clayson, Small Scale Processes in Geophysical Fluid Flows (Academic Press, San Diego, CA, 2000).

[6] R. W. Schmitt, Science 308, 685 (2005).

[7] T. Radko, Double-Diffusive Convection (Cambridge University Press, Cambridge, 2013).

[8] D. M. Anderson, G. B. McFadden, S. R. Coriell, and B. T. Murray, J. Fluid Mech. 647, 309 (2010).

[9] C. Karim, S. M. Jomaa, and A. Akbarzadeh, Solar Energy 85, 404 (2011).

[10] B. Huke and M. Lücke, J. Magn. Magn. Mater. 289, 264 (2005).

[11] M. C. Cross and P. C. Hohenberg, Rev. Mod. Phys. 65, 851 (1993).

[12] M. Lücke, W. Barten, P. Büchel, C. Fütterer, St. Hollinger, and Ch. Jung, in Lecture Notes in Physics, edited by F. H. Busse and S. C. Müller (Springer, Berlin, 1998), p. 127.

[13] A. Alonso, O. Batiste, A. Meseguer, and I. Mercader, Phys. Rev. E 75, 026310 (2007).

[14] P. Le Gal, A. Pocheau, and V. Croquette, Phys. Rev. Lett. 54, 2501 (1985).

[15] E. Moses and V. Steinberg, Phys. Rev. Lett. 57, 2018 (1986).

[16] T. Clune and E. Knobloch, Phys. Rev. A, 44, 8084 (1991).

[17] Ch. Jung, B. Huke, and M. Lücke, Phys. Rev. Lett. 81, 3651 (1998).

[18] S. Weggler, B. Huke, and M. Lücke, Phys. Rev. E 81, 016309 (2010).

[19] S. Rüdiger and F. Feudel, Phys. Rev. E 62, 4927 (2000).

[20] S. S. Leong, Numer. Heat Transfer, Part A 41, 673 (2002).

[21] K. Boronska and L. S. Tuckerman, Phys. Rev. E 81, 036320 (2010).

[22] K. Boronska and L. S. Tuckerman, Phys. Rev. E 81, 036321 (2010).

[23] I. Mercader, A. Alonso, and O. Batiste, Phys. Rev. E 77, 036313 (2008).

[24] A. Alonso, I. Mercader, and O. Batiste, Fluid Dyn. Res. 46, 041418 (2014).

[25] D. Henry and B. Roux, Phys. Fluids 30, 1656 (1987).

[26] P. Subramanian, O. Brausch, K. E. Daniels, E. Bodenschatz, T. M. Schneider, and W. Pesch, J. Fluid Mech. 794, 719 (2016).
[27] K. E. Daniels, B. B. Plapp, and E. Bodenschatz, Phys. Rev. Lett. 84, 5320 (2000).

[28] K. E. Daniels, O. Brausch, W. Pesch, and E. Bodenschatz, J. Fluid Mech. 597, 261 (2008).

[29] J. F. Torres, D. Henry, A. Komiya, and S. Maruyama, J. Fluid Mech. 756, 650 (2014).

[30] O. Shishkina and S. Horn, J. Fluid Mech. 790, 1 (2016).

[31] M. Ouriemi, P. Vasseur, and A. Bahloul, Numer. Heat Trans. A 48, 547 (2005).

[32] A. Bergeon, K. Ghorayeb, and A. Mojtabi, Phys. Fluids 11, 549 (1999).

[33] Z. W. Chen, Y. S. Li, and J. M. Zhan, Intnl. J. Heat Mass Transfer 55, 3633 (2012).

[34] F. Croccolo, F. Scheffold, and A. Vailati, Phys. Rev. Lett. 111, 014502 (2013).

[35] O. Sánchez, X. Ruiz, M. Pujalte, I. Mercader, O. Batiste, and J. Gavaldà, Intnl. J. Heat Mass Transfer 88, 508 (2015).

[36] O. Sánchez, I. Mercader, O. Batiste, and A. Alonso, Phys. Rev. E 93, 063113 (2016).

[37] I. Mercader, O. Batiste, and A. Alonso, Comput. Fluids 39, 215 (2010).

[38] S. Hugues and A. Randriamampianina, Int. J. Numer. Meth. Fluids 28, 501 (1998).

[39] B. Fornberg, A Practical Guide to Pseudospectral Methods (Cambridge University Press, Cambridge, 1998).

[40] L. N. Trefethen, Spectral Methods in Matlab (SIAM, Philadelphia, 2000).

[41] C. K. Mamun and L. S. Tuckerman, Phys. Fluids 7, 80 (1995).

[42] V. Frayssé, L. Giraud, S. Gratton, and J. Langou, Technical Report TR/PA/03/3, CERFACS; public domain software available at www.cerfacs.fr/algor/Softs.

[43] See Supplemental Material at http://link.aps.org/supplemental/ 10.1103/PhysRevE.97.023108 for Movie 1 which shows the time evolution of the contour plots of temperature fluctuation at $z=0.5$ for a SHC obtained for $\mathrm{Ra}=1610$. Movie 2 shows the significant states visited by the system during the formation of a SHC state when the mixture is initially homogeneous and a vertical temperature gradient is applied. Contour plots of temperature fluctuation at $z=0.5$ for $\mathrm{Ra}=1610$ are represented. Movie 3 shows the oscillations between a longitudinal roll state (LR) and a SHC state. Contour plots of temperature fluctuation at $z=0.5$ for $\mathrm{Ra}=1680$ are represented. 